\title{
Review on Aerodynamic Drag Reduction of Vehicles
}

\author{
A N M Mominul Islam Mukut and Mohammad Zoynal Abedin
}

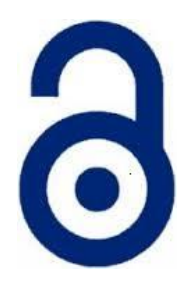

Received: 06 December 2018

Accepted: 22 December 2018

Published: 01 March 2019

Publisher: Deer Hill Publications

(c) 2019 The Author(s)

Creative Commons: CC BY 4.0

\begin{abstract}
Due to higher price, limited supply and negative impacts on environment by fossil fuel, automobile industries have directed their concentrations in reducing the fuel consumption of vehicles in order to achieve the lower aerodynamic drag. As a consequence, numerous researches have been carried out throughout the world for not only getting the optimum aerodynamic design with lower drag penalty and but also other parameters that increase the fuel consumption. In this regard, relevant experimental and numerical outcomes on vehicle drag reduction considering various techniques such as active, passive and combined techniques in order to delay or suppress flow separation behind the vehicles have been considered in this review paper. Furthermore, the effects of drag reduction and their applicability on the vehicles are also illustrated in this paper. Therefore, it is conjectured that the drag reduction has been improved as much as $20 \%, 21.2 \%$, and $30 \%$ by using the active, passive and combined control systems, respectively.
\end{abstract}

Keywords. Aerodynamic drag reduction, Flow control, Flow separation, Environmental impact, CFD

\section{INTRODUCTION}

Earlier time when fuel supply was not the main concern, cars were manufactured for high speed and stylish look ignoring the aerodynamic stability and fuel consumption. However, due to the crisis in fuel price and environmental impacts from exhaust emission, the researchers have focused their concentration to design the cars aerodynamically in order to reduce the fuel consumption as well as lowering the pollution. The drag which affects the fuel consumption can be minimized by designing the car with proper aerodynamic characteristics and also with implementation of lightweight materials for the body frame, which would decrease not only the fuel consumption but also the vehicle weight. However, most of fuel is consumed due to aerodynamic drag and the drag can be classified as skin friction drag and pressure drag. It is observed through an assessment that the pressure drag or form drag or parasite drag has the $80 \%$ contribution in the total aerodynamic drag due to the geometry of vehicles that leads to form the boundary layer separation and formation of wake region behind the vehicles [1]. Moreover, the car aerodynamic performances have been enhanced through the experimental and numerical approaches with extensive investigations [2-9]. Among these researches, it is seen that the methods of reducing drag and shape optimization are considered to be an inexpensive way to improve the fuel efficiency [9].

To improve the fuel consumption it is necessary to control flow separation at the rear of vehicles by applying flow control mechanisms that help either to delay or preventing the separation effect. The mechanism can be classified as active or passive flow control. The active flow control system includes movable body, holes or slots which are placed on the vehicle surface and their numbers also depend upon the flow control requirements. It is found that the active flow control system uses the actuator which consumes power from the vehicle [10]. Various systems like mechanical, electromagnetic, electric, piezoelectric or acoustic are used as the mechanism for the functioning of the system and it is observed that lower weight and smaller dimension are expected to reduce their negative impacts on vehicle performance [11]. On the other hand, conventional passive control systems consist of shape modification of vehicle or attachment of add-on devices to reduce the drag and it is seen that these arrangements of passive control systems show the significant advantages over the active control systems wherein the former systems are cheaper and consume no energy from the vehicles compared to the later systems [12].

\footnotetext{
A N M Mominul Islam Mukut and Mohammad Zoynal Abedin $\mathbb{x}$

Department of Mechanical Engineering

Dhaka University of Engineering \& Technology

Gazipur 1707, Bangladesh

E-mail: abedin.mzoynal@duet.ac.bd
}

Reference: Mukut, A. N. M. M. I. and Abedin, M. Z. (2019). Review on Aerodynamic Drag Reduction of Vehicles. International Journal of Engineering Materials and Manufacture, 4 (1), 1-14 
As the aerodynamic drag reduction and fuel consumption improvement are the main concern for the vehicles and the control mechanisms are necessary for the improved performance, various researches have been updated in these fields. Hence, it is necessary to list up all the updates and outcomes from the existing techniques and above all from the updated techniques which are eagerly awaited. Therefore, in the present review paper, it is analysed all the updates and outcomes from the existing techniques and above all from the updated techniques for the reduction of aerodynamic drag as well as for lowing the fuel consumption of the vehicles. The next section illustrates the aerodynamic drag impacts based on fuel consumption and their overall effects on environmental pollution.

\section{IMPACTS ON FUEL CONSUMPTION AND ENVIRONMENTAL POLLUTION}

Due to awareness regarding the environmental pollution, tremendous endeavours have been carried out in various sectors of automobile industries. Consequently, automobile industries are seen to manufacture the aerodynamically sound-vehicles that emit low emission as well as provide better fuel economy. It is revealed that aerodynamic drag varies with the square of the vehicle velocity, in which $70 \%$ of this drag are found to be accumulated from overall vehicle resistance at high speed [13]. It is also seen that reduction of vehicle aerodynamic drag is essential to lower greenhouse gas emission.

The amount of drag due to the vehicle shape i.e. pressure drag is the main contributor to total drag and it is noted that about $50 \%$ of vehicle's fuel consumption is due to aerodynamic drag at highway speeds [14]. Bellman et al. [15] extensively illustrated the analysis of reducing energy consumption of ground vehicles by active flow control. In these researches, it was also revealed that aerodynamic fuel efficient vehicles had good impact on fuel reserve as it could save 245 million gallons of fuel per year by improving 1\% in fuel economy. In addition, more than 30\% of $\mathrm{CO}_{2}$ and other greenhouse gas ( $\mathrm{GHG}$ ) emissions were seen to be occurred by these ground vehicles due to their fuel consumption. Moreover, most of the usable energy from the engine were responsible to overcoming the aerodynamic drag (53\%) and rolling resistance (32\%); only $9 \%$ were required for auxiliary equipment and $6 \%$ were used by the drive-train. It was also found that $15 \%$ reduction in aerodynamic drag at highway speed of $55 \mathrm{mph}$ could result in about $5-7 \%$ in fuel saving. Due to these numerous findings, researches on vehicles aerodynamic drag reduction have seen to be prime issue in the scientific community by using different flow control techniques.

\section{IMPACT OF ACTIVE FLOW CONTROL}

The active flow control is a mechanism that modifies the flow field in the wake region in order to reduce the aerodynamic drag by increasing static pressure. The pressure losses in the wake region has a great contribution to increase the aerodynamic drag, and hence, the penalty is maximum for square back vehicles. This could be minimized by proper designing of rear shape of the vehicles but there are limited options in this regard for automobile industries due to the customer demand in the case of outlook appearance, ergonomically design and stability of the vehicle. Moreover, this could be achieved by introducing active flow control techniques which can alter the flow structure behind the vehicles without physical modification of vehicles. Several active flow control techniques used for reducing vehicle aerodynamic drag have been investigated both numerically and experimentally [16-39]. The main advantage of this type of active control technique is attributed to be a method of quick response in a way that there is no externally added devices on the vehicles' body which causes no penalty of parasite drag. In this method, the flow can be controlled actively by movable parts, synthetic jet, steady blowing, suction, plasma actuator of having some unique features.

\subsection{Movable Underbody Diffuser}

The movable underbody diffuser technology is used for reducing aerodynamic drag in the vehicle by controlling rear flow field. For instance, it is extensively revealed that the rear flow field of vehicle is influenced by the flow coming out under the vehicles which implies that the rear flow field can be modified by controlling the flow under the vehicles [16, 17]. Kang et al. [18] developed a movable under body diffuser as shown in Figure 1 and Figure 2, and numerically investigated that the automobile's aerodynamic drag could be reduced by an average of more than $4 \%$, which would help to enhance the constant speed fuel efficiency by approximately $2 \%$ at a range of driving speeds exceeding $70 \mathrm{~km} / \mathrm{h}$.

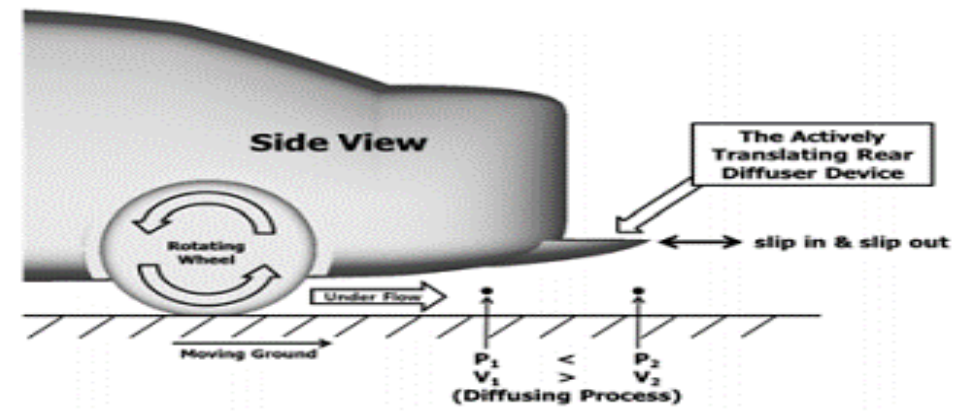

Figure 1: Basic concept of actively translating rear diffuser [18]. 


\subsection{Steady Blowing}

Vehicles' aerodynamic characteristics have been greatly influenced by the shape of rear end, which also affects the stability and comfort. The effect of steady blowing has been investigated experimentally on a realistic car model by Heinemann et al. [19], at three different position namely, (i) perpendicular top position air jet (ii) perpendicular bottom position air jet (iii) tangential bottom position air jet which are shown in Figure 3. Their results showed that rear axle lift was reduced by about $5 \%$ with coefficient of drag (CD) changes around $1 \%$. Another investigation has been carried out to evaluate the effectiveness of steady blowing on the wake structure of a simplified $1 / 4$ scale square back vehicles at a variety of angles on the roof trailing edge [20]. Its effects were evaluated by implementing various flow and pressure measurement techniques and results showed that overall gains were achieved. But due to the requirement for large mass flow rate, this technique has limited applicability to road vehicles. The influence of steady blowing has also been investigated by both experimentally and numerically on an Ahmed body at $25^{\circ}$ slant angle and depending on Reynolds's number, and the drag reduction has been achieved from 6 to $10.4 \%$ [20, 21], 6.4\% [22], $5.7 \%$ and $2.2 \%$ (at $90^{\circ}$ slant angle) [23], respectively. The effect of blowing angle has been investigated numerically and it is found that $11.1 \%$ drag reduction has been obtained at a blowing angle $45^{\circ}$ [24].

An array of blowing steady 53 micro jets as shown in Figure 4 were also found to be effective on a model of a generic vehicle shape, the Ahmed body with a $25^{\circ}$ slant in reducing drag coefficient (9-14\%) and lift coefficient (up to $42 \%$ ) depending on Reynolds Number [25]. These arrays of steady micro jets were positioned at $6 \mathrm{~mm}$ downstream of the separation line between the roof and the slanted rear window. The use of small scale, steady jets (micro jets) in normal and tangential injection orientations has been investigated through experimental parametric studies and compared with numerical simulations on Honda simplified Body (HBS) and it is found that drag experienced by HSB is reduced by nearly $2.6 \%$ with net reduction in power consumption [26].

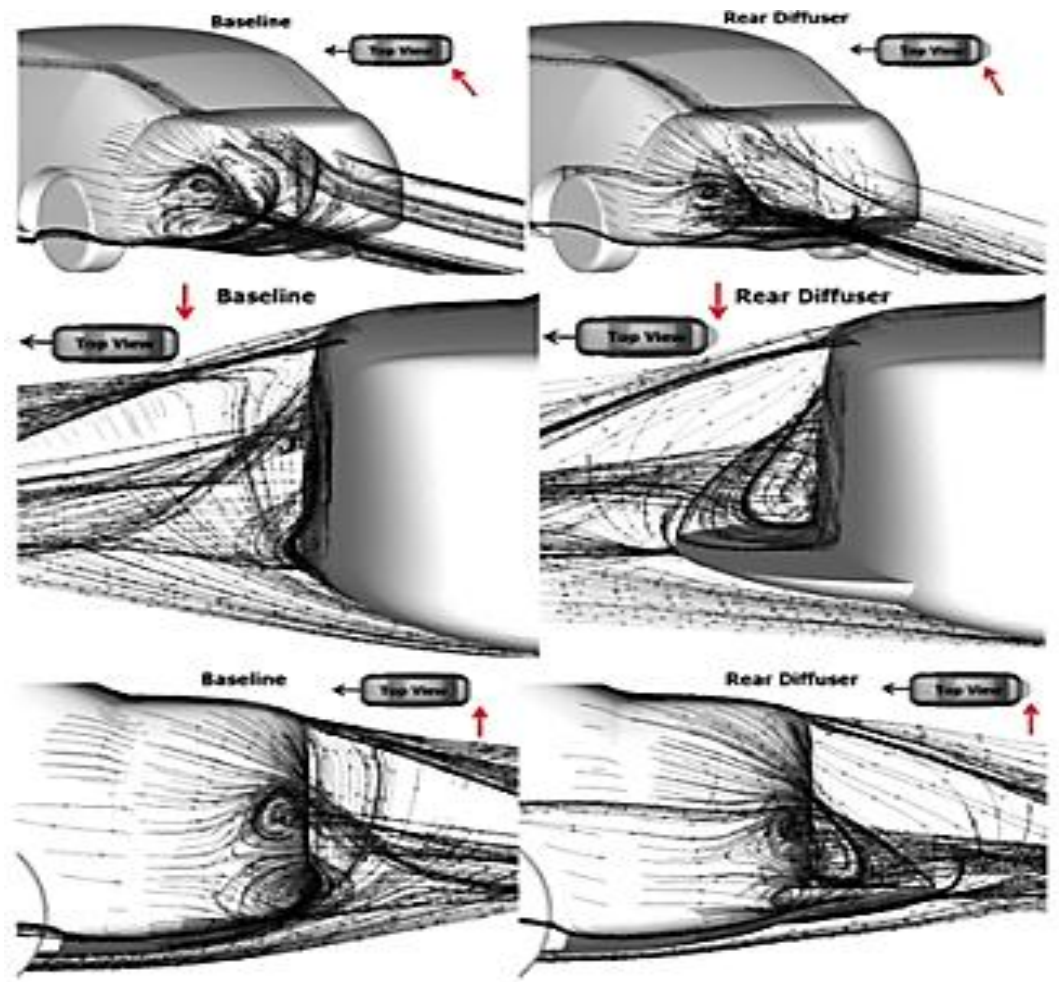

Figure 2: Effectiveness of actively sliding diffuser at three different angles [18].

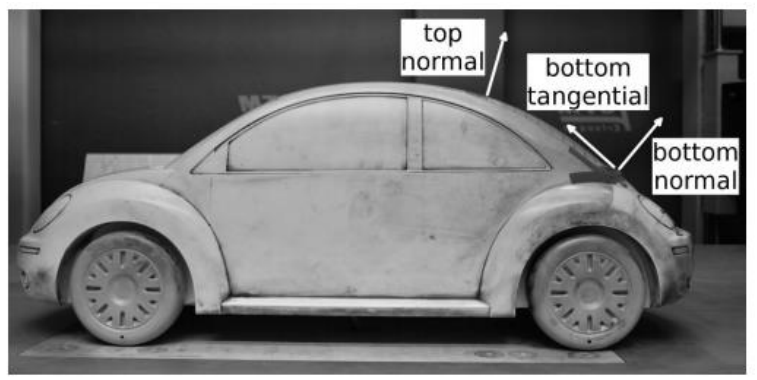

Figure 3: Positions of blowing jets [19]. 


\subsection{Synthetic Jets}

Synthetic jet actuator is generally formed by orifice, cavity and piezoelectric actuator or diaphragm where flow moves back and forth through a small opening by the movement of diaphragm. An experimental parametric study has been carried out by using an array of 12 synthetic jets on an Ahmed model at two different slant angles and it is revealed that the aerodynamic drag is seen to be reduced to $4.29 \%$ for $25^{\circ}$ slanted body, but increased for $35^{\circ}$ model [27]. Several experimental works have been carried out with synthetic actuator jets on an Ahmed body as shown in Figure 5 and it is found that maximum drag reduction depends on Reynolds's number which is equal to $8.5 \%$ for $\operatorname{Re}=1.2$ $\times 10^{6}$ and $6.5 \%$ for $\operatorname{Re}=1.9 \times 10^{6}[28]$ and $10 \%$ for $\operatorname{Re}=2.3 \times 10^{6}[29]$.

\subsection{Pulsed Jet}

Effectiveness of pulsed jet has been investigated experimentally on the suppression of the rear slant recirculation bubble, without acting on longitudinal structures on an Ahmed body with $25^{\circ}$ slant angle and it is found that $6-8 \%$ maximum drag reduction is obtained depending on the geometric and jet exhaust configurations that show different sensitivity to the forcing parameters [30]. Another investigation has been carried out with an Ahmed body having $35^{\circ}$ slant angle where $20 \%$ drag reduction is achieved that confirms the interest in using pulsed jets (shown in Figure 6) in order to reduce aerodynamic drag and pollutant emission [31]. By acting on the kinematic of the wake, this control technique changes the wall pressure and shear stress distributions on geometry of vehicles and generates evolutions in energy dissipation per unit process [32].

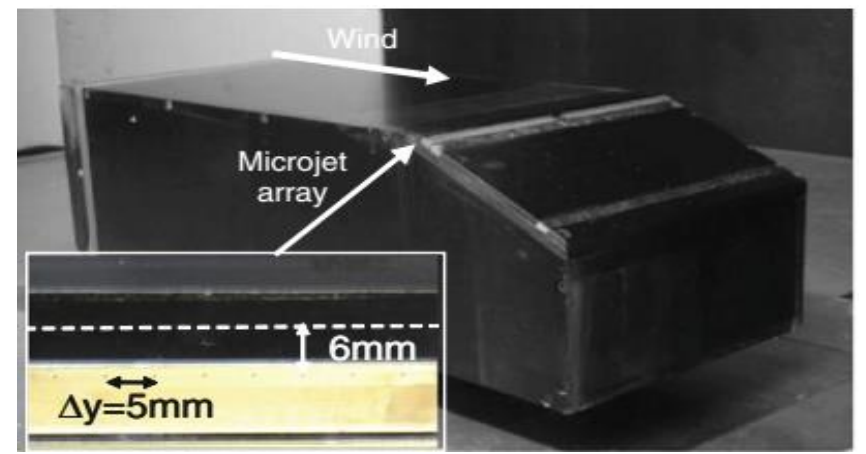

Figure 4: Steady micro jet actuator array mounted on the rear, slanted window of the Ahmed body [25].

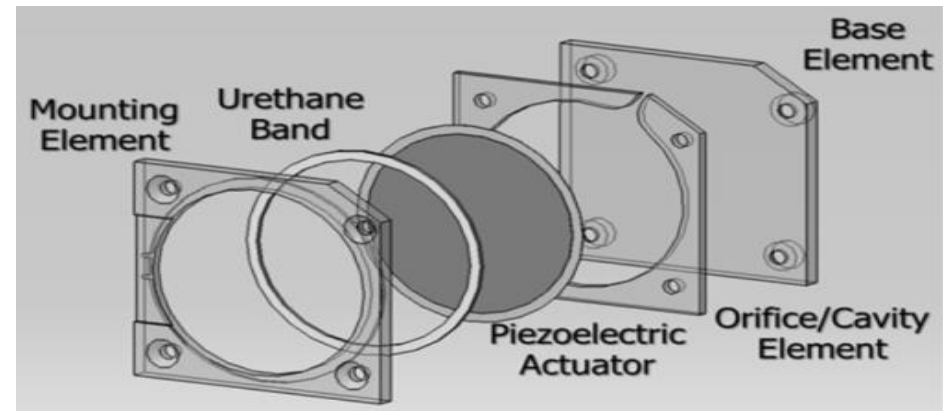

Figure 5: Components of a synthetic jet actuator [28].

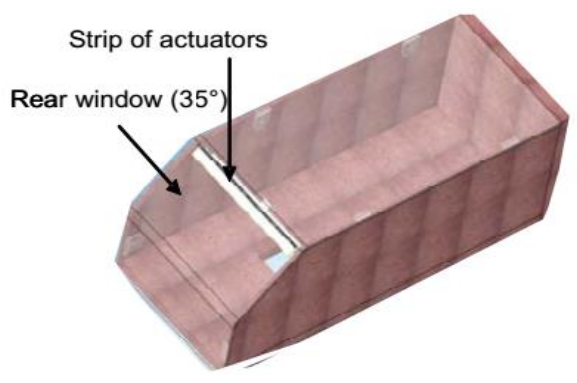

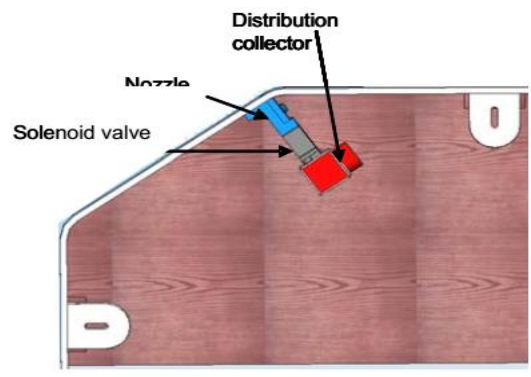

b

Figure 6: (a) Locations of actuator on the Ahmed body and (b) Longitudinal section with a view of the implementation of the pulsed jets [31]. 


\subsection{Steady Suction}

Automobile industries seek better solution to reduce pollution and fuel consumption, in this regard, active flow control techniques have got huge attention as it requires no shape modification of the vehicles. Steady suction is an active flow technique which modify vortices that affects rear wake of the vehicle. The workability of steady suction has been evaluated by both experimental and numerical approaches on simplified fastback car geometry as shown in Figure 7 and this technique is capable to suppress rear window separation and reduce drag more than 17\% [33]. The contribution of this method towards separated region has been analysed by flow topology on an iso-surface of total pressure loss, which is shown in Figure 8. It is found that suction is effective to eliminate the separated layer developed and reattachment of flow is obtained. The effect of suction around the lateral edges of a simplified car windshield has been tested experimentally in a water tunnel [34], which provides a drag reduction of $6 \%$.

A Large Eddy Simulation (LES) has been carried out on a $25^{\circ}$ slant angle on an Ahmed body, in a view to that the combined effect is advantageous over single alone as net zero mas flux [35]. In their studies, twelve slits were arranged in six pairs in which each pair provides blowing and suction as shown in Figure 9 and a drag reduction of $9.5 \%$ has been found. The combined control of suction and blowing is seen to be effective on changing the rear window wake structure, in which streamlines of time average flow is separated (without control) and re-attached (with combined control) as shown in Figure 10. Another experimental and numerical studies have been conducted to evaluate the suction and blowing control on drag reduction on an Ahmed body in three different ways namely, (i) only suction, (ii) only blowing and (iii) combination of both control [36]. The outcomes show that their combined effect enhances the drag reduction which is shown in Figure 11 and an average of $10 \%$ drag reduction is recorded.

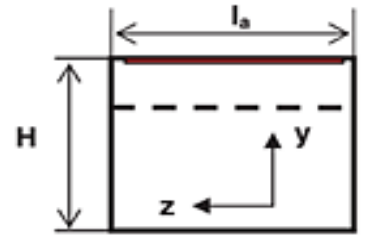

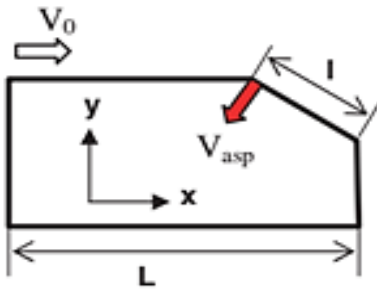

$\mathbf{L}$

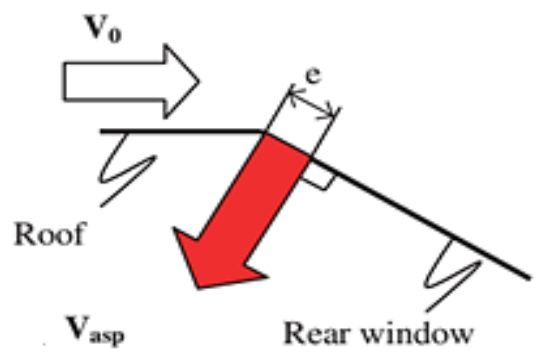

b

Figure 7: (a) Schematic of the used geometry and (b) Implementation of the control system [33].

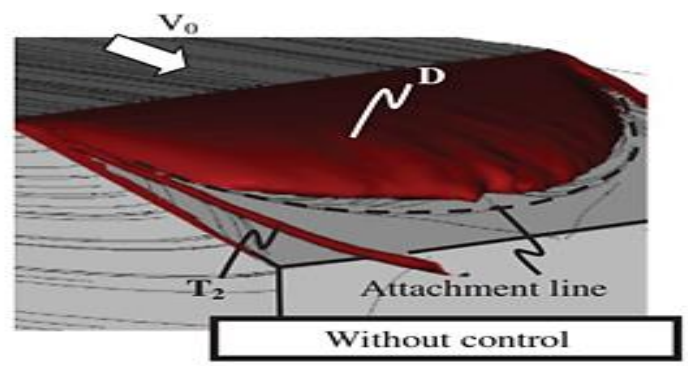

a

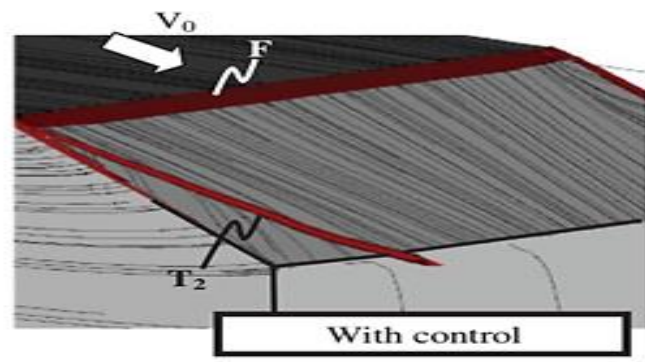

b

Figure 8: Effects of suction on rear window (a) without control and (b) with control [33].
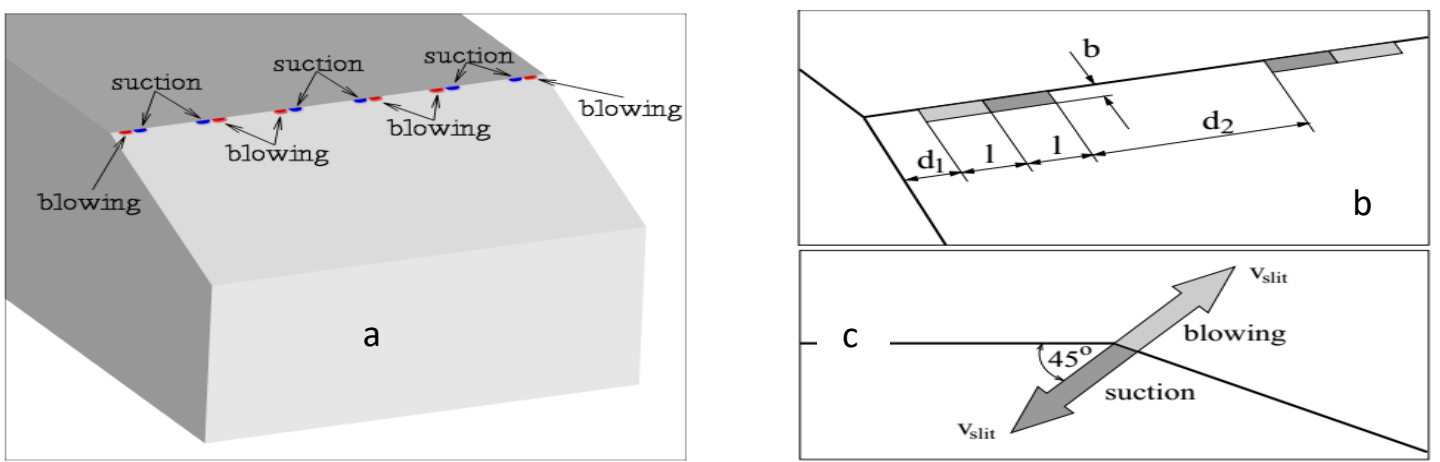

Figure 9: Arrangement of slits (a) Along the upper rear edge, (b) Slit positions and size, and (c) Direction of Blowing/suction [35]. 


\subsection{Plasma Actuator}

Plasma actuator (PA) is another effective means of active flow control due to its some advantages such as easier to construct, quick to response, requires no pump and holes. The performance of PA has been investigated experimentally on an Ahmed body with $25^{\circ}$ slant angle where 10 PAs are used, among of them, 6 PAs are placed on the separation region on the rear wind and four are placed in two-two pairs in the region where two longitudinal vortices are observed from both sides of the rear window [37]. These position of PAs are actually based on the visualization of the topological structures of the skin friction field using oil film which is shown in Figure 12. The research shows that PAs have effective outcomes on suppressing the separation at the rear end of vehicles and $8 \%$ drag reduction is obtained.

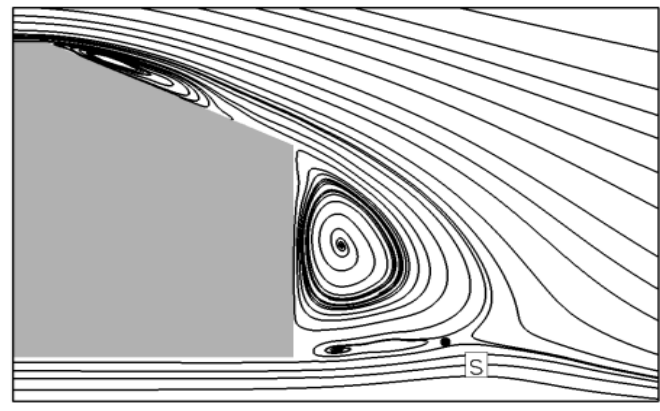

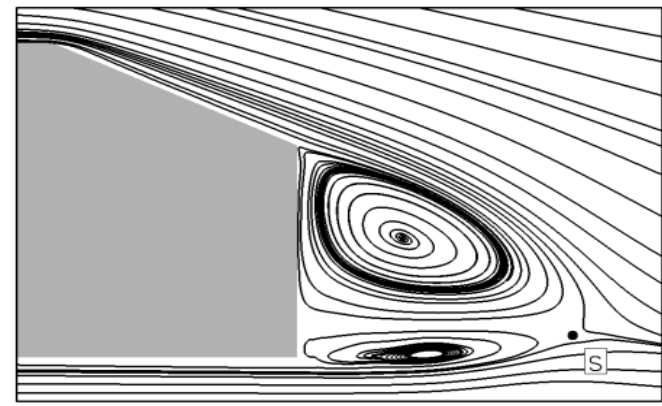

Figure 10: Streamlines of the time-averaged flow in the symmetry plane, (a) $y=0$, for the reference flow and (b) the controlled flow; location of saddle point is marked [35]

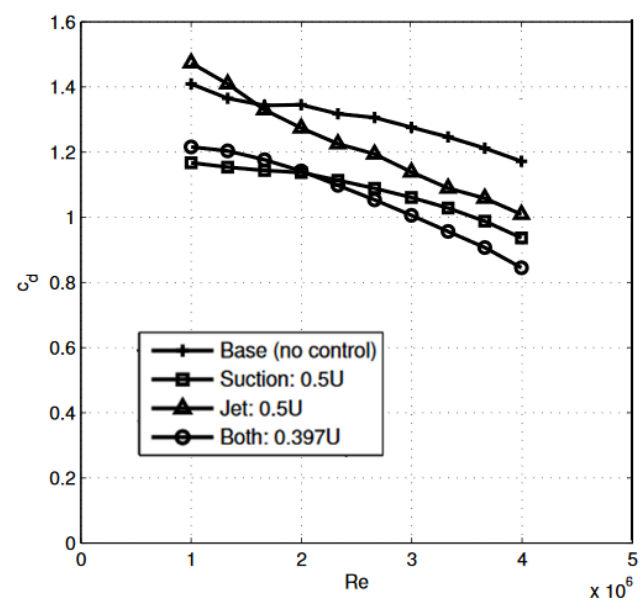

Figure 11: Effects of actuator on drag reduction [36].

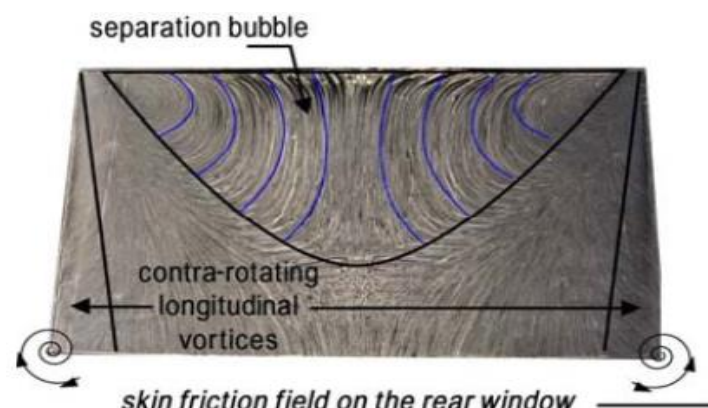

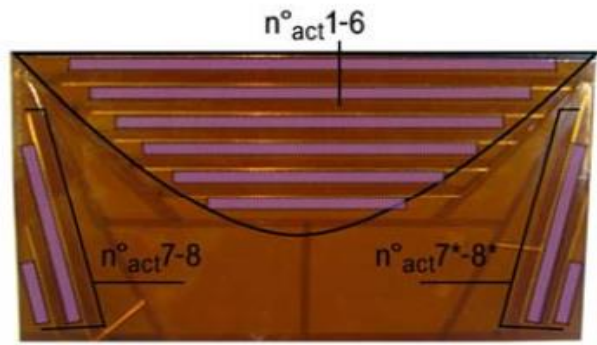

location of $D B D$ actuators

Figure 12: (a) Separation zone and (b) Location of ten DBD actuators relatively to skin friction field on the rear window [37]. 
Another experimental investigation with single PA shows that it can reduce drag force in steady and unsteady actuations by $3.65 \%$ and $2.44 \%$, respectively [38]. A series of experiments have been carried out on an Ahmed body having $0^{\circ}$ and $35^{\circ}$ slant angles and each of these are further modified by rounding the edges with different radii at different air velocity [39]. In this study, it is found that $20 \%$ drag is reduced for a velocity below $15 \mathrm{~m} / \mathrm{s}$, and $3 \%$ reduction is obtained at the highest tested velocity of $20 \mathrm{~m} / \mathrm{s}$. It is concluded that in order to increase the drag reduction at a higher velocity it is required to maintain higher voltage in PA which is not possible as the models used are so small to be withstand with higher voltage.

\section{IMPACT OF PASSIVE FLOW CONTROL}

The passive flow control system needs extra add-on device/devices which are attached permanently on the vehicle surface where flow control is necessary. Although the active flow control technique is easier to construct and employ on the vehicle body, but it consumes energy from the vehicles, in that regard, passive flow control technique would be better alternative if this technique is properly applied on the vehicles. The following sections describes the different passive flow control techniques.

\subsection{Vortex Generator}

Vortex generators (VG) is an aerodynamic body permanently fixed on vehicle with a certain angle with flow direction. Generally, VGs are placed in a group to create clock-wise or counter-clockwise vortices (as per their configuration) which add momentum to near wall surface where flow is separated, as a result, the separation is suppressed or shifted further downstream. An extensive experimental studies have been carried out to evaluate the performance of VGs on aerodynamic drag reduction [40]. In this research, three combinations of VGs namely, (i) complete line-22 VGs (ii) 4 VGs on each side (iii) 14 VGs in the centre were used on a modified Ahmed body which is shown in Figure 13. Details of flow structure are captured by PIV and hot-wire anemometry. Parametric studies of VG has also been conducted to figure out the optimum outcome by considering the effect of angle of VG with flow direction, their placement, effect on Re. Among of the tested done with three configurations of VGs, complete line of vortex generators is the most effective as it reduces the drag and lift as $12 \%$ and $60 \%$, respectively.

The performance of a special type of VG known as pocket type VG has been investigated numerically on a minivan [41]. The aim of using this type of VG is to avoid additional drag that is arisen from then conventional VGs, as these are attached externally to the vehicle body. A pair of pocket type VGs are placed in the left and right side of the rear roof end of the Low Mass Vehicle (LMV) to evaluate the effectiveness which is shown in Figure 14. The outcome shows that it can reduce the drag of $2.2 \%$.

An experimental investigation has been carried out on an Ahmed body with $25^{\circ}$ slant angle at a free stream velocity of $20 \mathrm{~m} / \mathrm{s}$ using cylindrical shape VGs [42]. Among the tested various configuration of VGs, maximum drag reduction of $10 \%$ is found. A slight larger drag reduction (11.7\%) is found in a numerical investigation of cylindrical shape VGs [43]. The effectiveness of VGs are also evaluated numerically on SUV and Ahmed body with a drag reduction of $4.2 \%$ and $10 \%$, respectively [44]. On the other hand, an experimental and numerical investigation has been carried out on a sedan car using 3 delta type of VGs at different yaw angles [45], where the middle VG was kept stationary and remaining two were changed with their angle using smaller stepper motor as shown in Figure 15. In the research, a maximum reduction of drag and lift coefficient have been found as $4.53 \%$ and $2.55 \%$, respectively.

In support of drag reduction by VGs, different turbulence model named as LES, DES and RANS have been used in an open source computational fluid dynamics (CFD) of the Ahmed body with a $25^{\circ}$ slant angle [46]. The results are well validated with the experimental works done for 3D bluff body using coherent stream wise streaks [42].

\subsection{Spoiler}

A spoiler is an external structure attached to the rear end of the vehicles to control the flow at downstream that helps to minimize the turbulence behind the vehicle and also append downward pressure which reduces the lift. The influence of spoiler on vehicle aerodynamics has numerically investigated on a Toyota Eco car model as shown in Figure 16, and it is found that by using this spoiler at a speed of $30 \mathrm{~m} / \mathrm{s}$, drag and lift, respectively can be reduced by $5 \%$ and more than $100 \%$ [47].

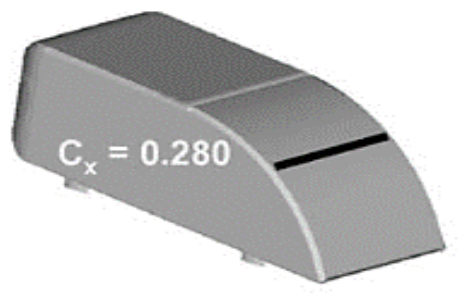

Complete line

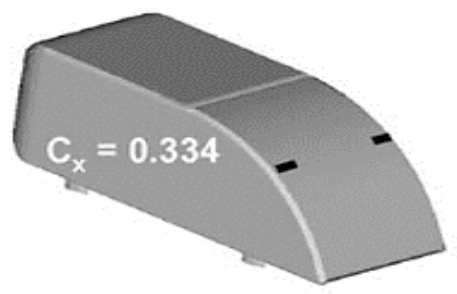

4 VG on each side

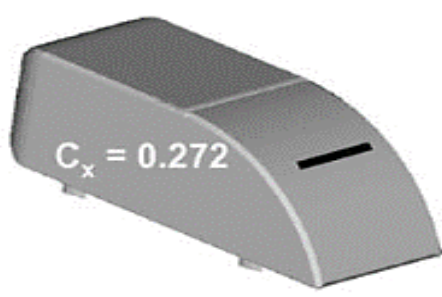

14 VG in the center

Figure 13: Sketch of three configurations of VGs [40]. 


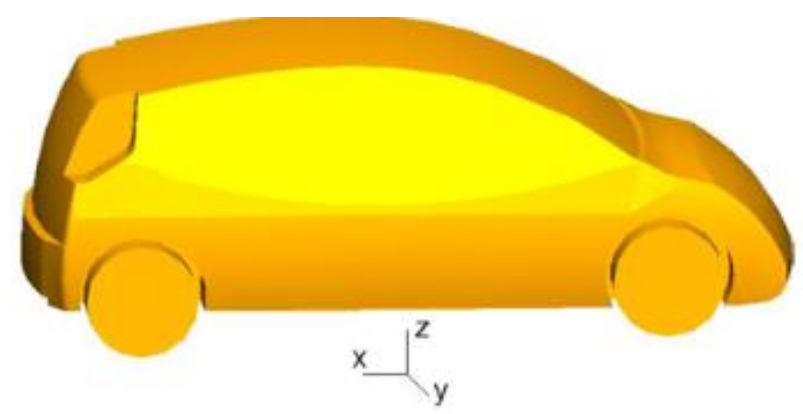

Figure 14: Surface geometry of the Low Mass Vehicle (LMV) with a pair of pockets [41].

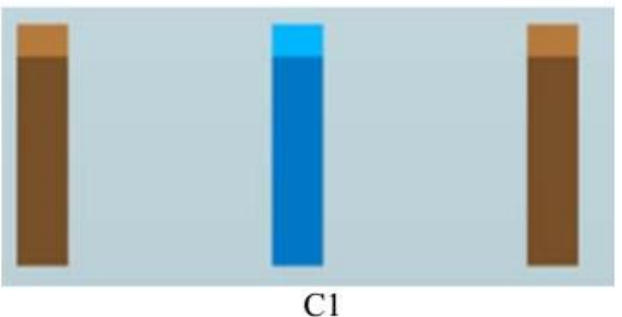

C1

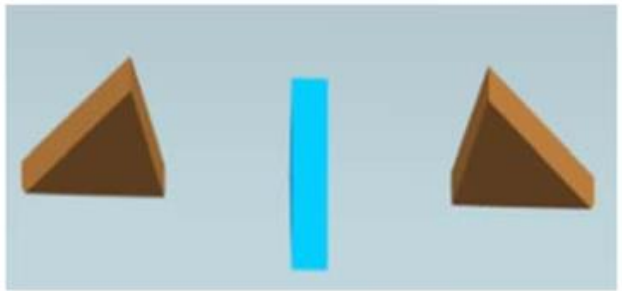

$\mathrm{C} 3$

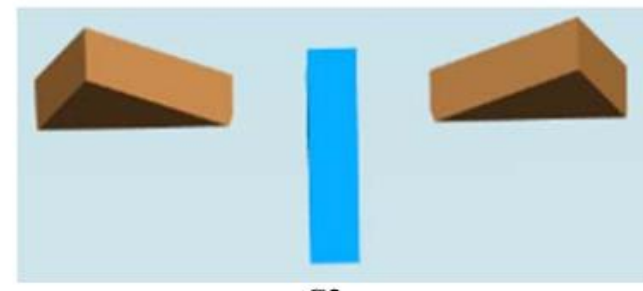

$\mathrm{C} 2$

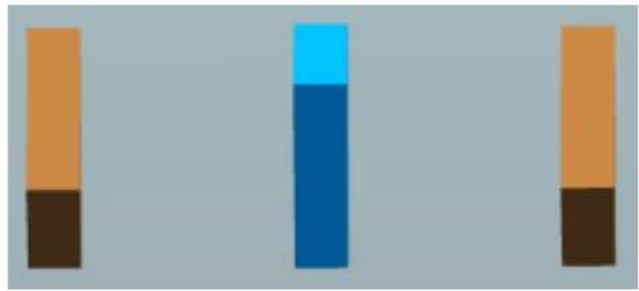

$\mathrm{C} 4$

Figure 15: Four different configurations of three VGs [45].

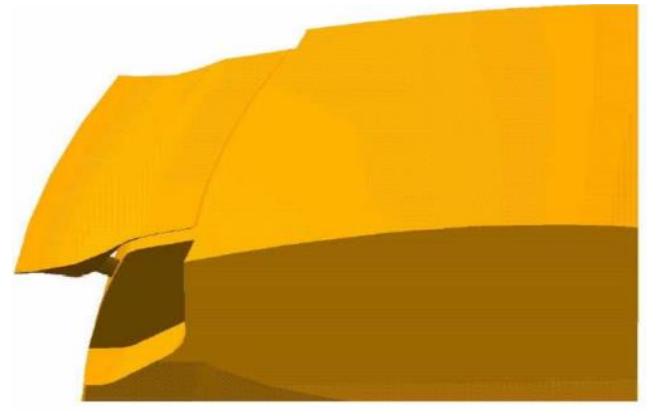

a

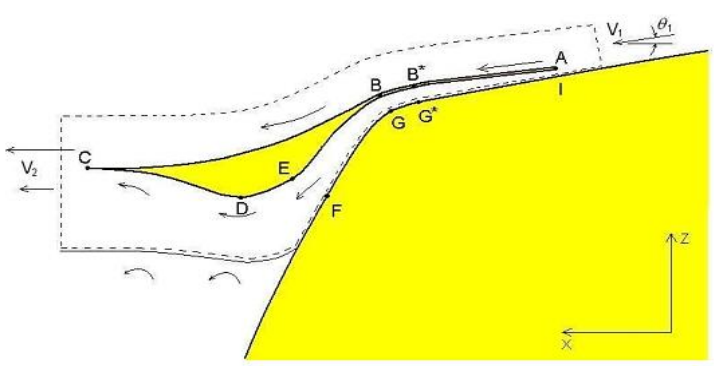

$b$

Figure 16: Location of spoiler attached to the back of a modified LMV (a) 3D view and (b) Cross-sectional view [47].

\subsection{Flaps}

Flaps are another extended surfaces which are attached with vehicle body to reduce the drag and altering the flow at the rear of vehicles to enhance aerodynamic performances. The performance of flaps has been investigated with three different free stream velocities of $20 \mathrm{~m} / \mathrm{s}, 30 \mathrm{~m} / \mathrm{s}$ and $40 \mathrm{~m} / \mathrm{s}$, by placing two flaps on the side edges of the rear slant and two flaps on the top of the rear slant, and hence the reduction of drag has been found as much as $17.6 \%$ and $15 \%$, respectively for the two arrangements of flaps [48]. A special type of flap called Automatic Moving Deflector (AMD) taking inspiration from birds had been tested experimentally on an Ahmed body as shown in Figure 17 , by which the maximum drag reduction is achieved from the pressure recovery by AMD which is 19\% [49].

Another numerical investigation has been carried out with configuration of flap structures at the four edges of slant surfaces for the angles of $80^{\circ}, 40^{\circ}$ and $20^{\circ}$ in which the values of drag reduction are found as $21.1 \%, 21.2 \%$ and $17.9 \%$, respectively [50]. 


\subsection{Body Modification}

Body modification is the passive technique to enhance aerodynamic drag reduction which helps to reduce fuel consumption. Body modification includes vehicle body outline, front and rear part; underbody geometry of the vehicle, sometimes extra added surfaces or diffusers which alter the bottom geometry of vehicles. These diffusers actually increase the air velocity below the vehicles which reduce the air pressure. As a result vehicles stability is improved as the downward forces are increased. The bottom of a Sedan and Wagon had been modified which has an angle at the bottom rear that acts like an underbody diffuser [51]. The performance of underbody diffuser has been tested with varying angles shown in Figure 18. Results from this study showed a potential aerodynamic drag reduction of the sedan car approximately $10 \%$, and the wagon car by $2-3 \%$.

The effect of underbody diffuser with flaps and vanes has been numerically investigated on a formula SAE car [52]. The addition of vanes with diffuser enhanced the pumping capacity of diffuser as well as increase up to $13 \%$ down force. The addition of a flap above the trailing edge of the diffuser also increased down force by $25 \%$. A numerical investigation has been carried out with different underbody drag reduction devices like undercover, underfin, and side air dam on the actual shape of a sedan-type vehicle [53], among these underbody has the ability to reduce the aerodynamic drag by $8.4 \%$. Shape optimization has been carried out on a sedan car by Artificial Neural Network (ANN), which focus on rear shape modification [54]. To achieve the aerodynamic goal by shape optimization, six local parts from the end of sedan have been chosen as design variables and an ANN approximation model was established with 64 experimental points generated by the D-optimal methodology. Due to these shape optimization, 5.639\% lower coefficient of drag has been obtained. Six parameters namely, (i) hood (ii) windshield (iii) rear window (iv) side window ( $v$ ) rear-end shrinkage and ( $v i$ ) trunk lid have been modified to optimize body shape numerically on a simplified sedan car [55]. A reduction of $13.23 \%$ aerodynamic drag has been achieved. Numerical investigation has been carried out on an Ahmed body with non-smooth dimpled surfaces on its slant back [56]. The geometry of non-smooth surfaces are shown in Figure 19. In order to maximize the drag reduction performance of the dimpled non-smooth surface, an aerodynamic optimization method based on a Kriging Surrogate model was employed to design the dimpled non-smooth surface. Four structure parameters had been selected as the design variables, and a 16-level design-of experiments method based on orthogonal arrays had been used to analyses the sensitivities and the influences of the variables on the drag coefficient; a surrogate model had been constructed from these. The results showed that the optimal combination of design variables can reduce the aerodynamic drag coefficient by $5.20 \%$.

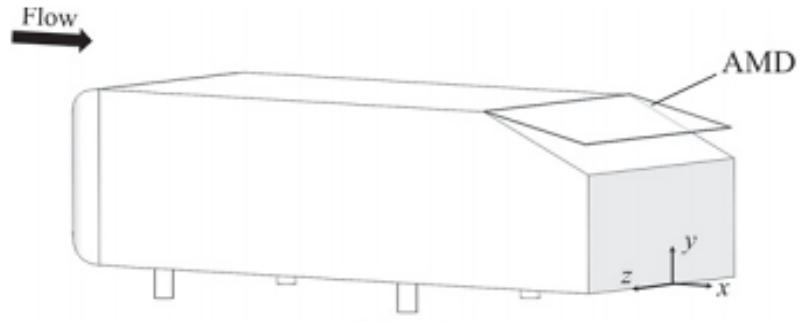

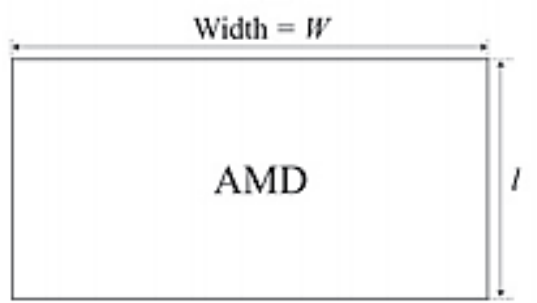

b

Figure 17: Details of AMD (a) attachment on Ahmed body and (b) AMD configuration [49].

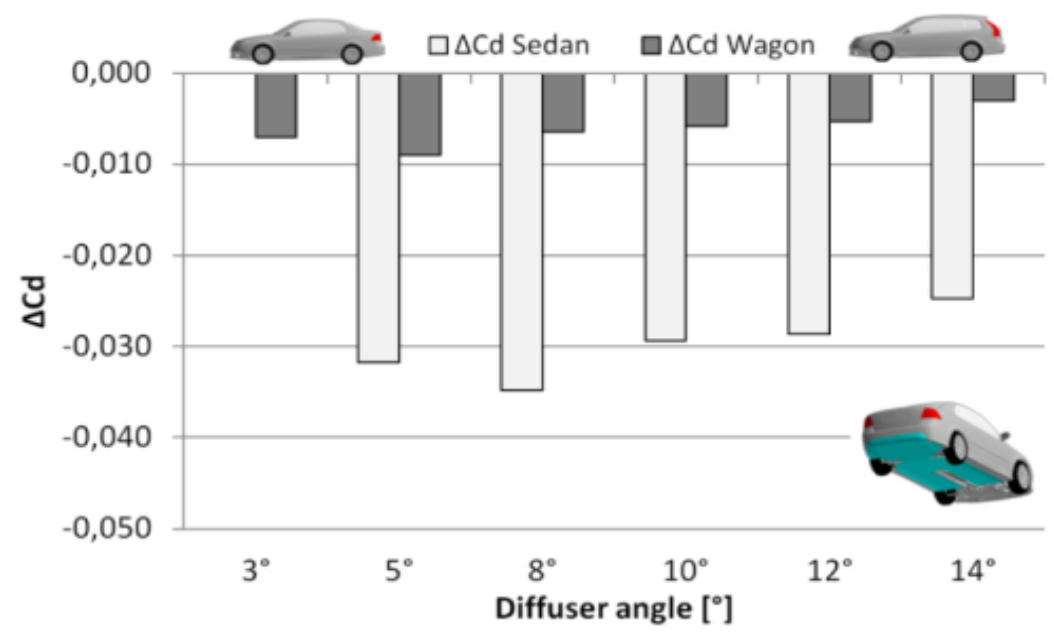

Figure 18: Comparison of drag coefficient for Sedan and Wagon [51]. 


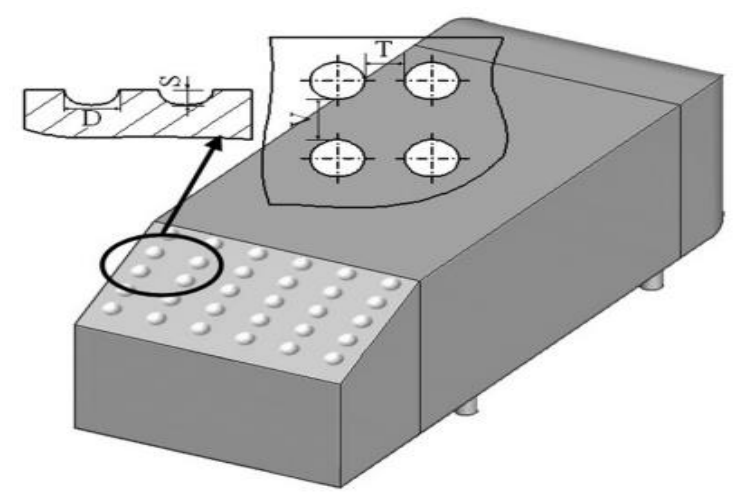

Figure 19: Non-smooth dimpled surfaces [56].

\section{IMPACT OF COMBINED FLOW CONTROL SUMMARY}

Several flow control techniques have been investigated and implemented to reduce aerodynamic drag which is the main contributor to increase fuel consumption. Each techniques has its unique advantages and disadvantages over each other. To achieve the best, combination of more than one technique would bring more advantages to obtain the desire drag reduction. The combination of blowing jets as active flow control and porous layer as passive flow control were implemented on an Ahmed body and results showed that a drag reduction of $30 \%$ could be achieved [57].

\section{SUMMARY}

Various flow control methods are discussed with their outcomes in the paper. Some methods are applied individually and some are applied combined. To figure out the effectiveness of each control methods to reduce aerodynamic drag reduction, it would be better to represent them in a table with some graphical representation. A summary of drag reduction using various flow control techniques is shown in Table 1. Among various methods, it is found that combined control technique is more beneficial as it can reduce maximum drag reduction than single method.

Percentage of drag reduction by active and passive methods are graphically presented in Figure 20 and Figure 21 respectively. Maximum contribution of drag reduction for different control methods is shown in Figure 22. From the above analysis, it is seen that the drag reduction for active control system could be as much as $20 \%$ by using the pulse jet [31, 32] and plasma actuator [39] as shown in Figure 20 and 22. On the other hand, for passive control system, the drag could be decreased to $21.2 \%$ by using Flaps [50] as shown in Figure 21 and 22. However, for combined flow control technique, the drag could be reduced as much as $30 \%$ by using blowing jets with porous layer [57] as shown in Figure 22.

Table 1: Summary of flow control methods on aerodynamic drag reduction

\begin{tabular}{|c|c|c|}
\hline Types of control & Name of applied method & Percentage of drag reduction [Reference] \\
\hline Active Flow Control & $\begin{array}{l}\text { Movable underbody diffuser } \\
\text { Steady blowing } \\
\text { Synthetic Jets } \\
\text { Pulsed Jet } \\
\text { Steady suction } \\
\text { Plasma Actuator }\end{array}$ & $\begin{array}{l}4 \%[18] \\
1 \%[19], 6 \text { to } 10.4 \%[20,21], 6.4 \%[22], 5.7 \%[23], \\
11.1 \%[24], 9-14 \%[25], 2.6 \%[26] \\
4.29 \%[27], 8.5 \%\left(\operatorname{Re}=1.2 \times 10^{6}\right) \text { and } 6.5 \%(\operatorname{Re}=1.9 \\
\left.\times 10^{6}\right)[28] \text { and } 10 \%\left(\operatorname{Re}=2.3 \times 10^{6}\right)[29] \\
6 \text { to } 8 \%[30], 20 \%[31] \text { and }[32] \\
17 \%[33], 6 \%[34], 9.5 \%[35], 10 \%[36] \\
8 \%[37], 3.65 \%[38], 20 \%[39]\end{array}$ \\
\hline Passive Flow Control & $\begin{array}{l}\text { Vortex Generator } \\
\text { Spoiler } \\
\text { Flaps } \\
\text { Body modification }\end{array}$ & $\begin{array}{l}12 \%[40], 2.2 \%[41], 10 \% \text { [42] and [46], 11.7\% [43], } \\
4.2 \% \text { (SUB) and 10\% (Ahmed Body) [44], 4.53\% [45] } \\
5 \%[47] \\
17.6 \%[48], 19 \%[49], 21.2 \%[50] \\
10 \%[51], 8.4 \%[53], 5.639 \%[54], 13.23 \%[55], \\
5.20 \%[56]\end{array}$ \\
\hline $\begin{array}{l}\text { Combined } \\
\text { Control }\end{array}$ & $\begin{array}{l}\text { blowing jets with porous } \\
\text { layer }\end{array}$ & $30 \%[57]$ \\
\hline
\end{tabular}




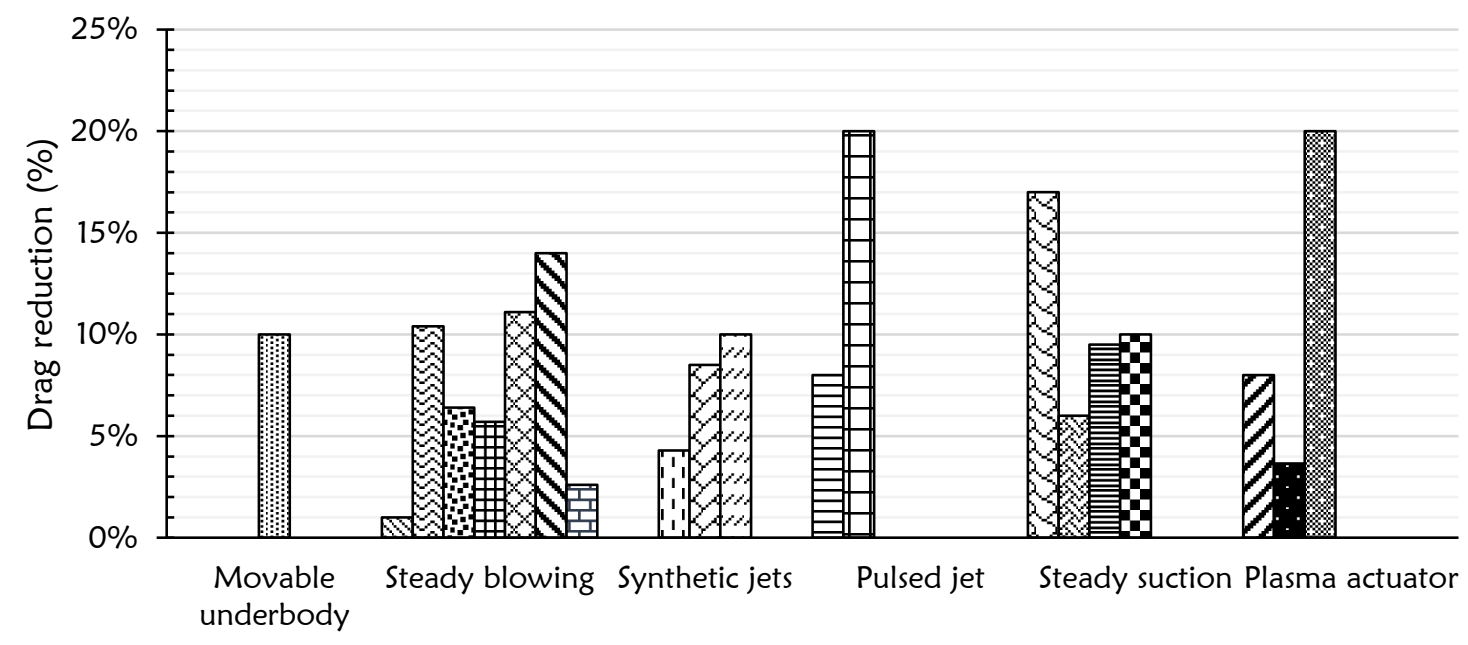

Active control methods

\begin{tabular}{|c|c|c|c|c|c|c|c|c|c|}
\hline $\begin{array}{l}\text { Bar } \\
\text { chart }\end{array}$ & Reference & $\begin{array}{l}\text { Bar } \\
\text { chart }\end{array}$ & Reference & $\begin{array}{l}\text { Bar } \\
\text { chart }\end{array}$ & Reference & $\begin{array}{l}\text { Bar } \\
\text { chart }\end{array}$ & Reference & $\begin{array}{l}\text { Bar } \\
\text { chart }\end{array}$ & Reference \\
\hline 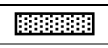 & [18] & 回覀田 & [23] & {$[1,1,1$} & [27] & 田里 & [31] & Exy & [36] \\
\hline \$\$ & [19] & 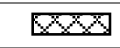 & {$[24]$} & $\overline{\angle Y Y}$ & [28] & ळब & [33] & $Z Z Z$ & [37] \\
\hline$\propto 2$ & {$[20,21]$} & 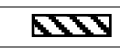 & [25] & 世COA & [29] & 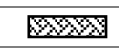 & [34] & E::::? & [38] \\
\hline EFPOA & [22] & 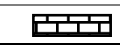 & [26] & ए & [30] & 原 & [35] & \% & [39] \\
\hline
\end{tabular}

Figure 20: Percentage of drag reduction by active control methods.

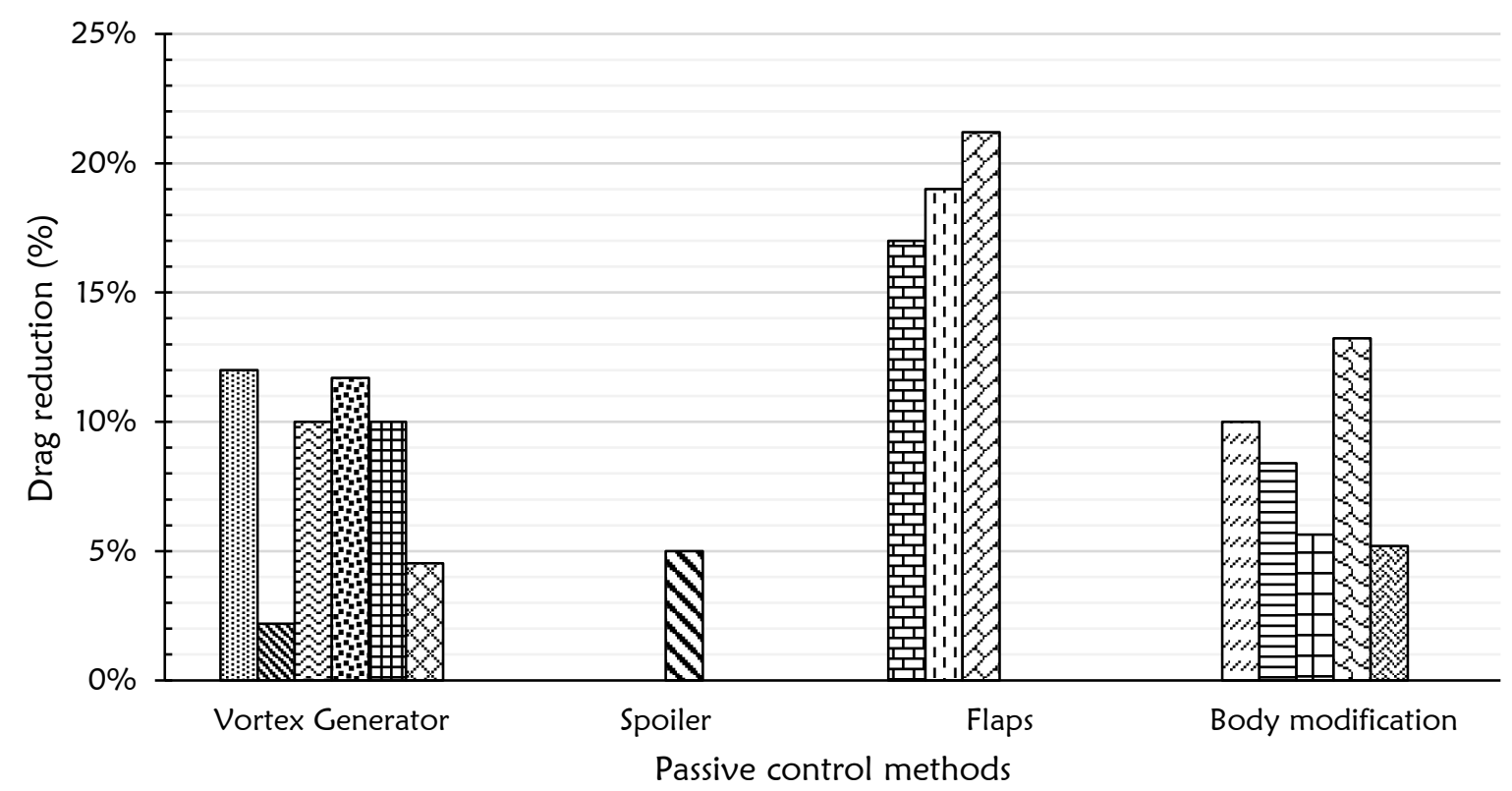

\begin{tabular}{|c|c|c|c|c|c|c|c|}
\hline Bar chart & Reference & Bar chart & Reference & Bar chart & Reference & Bar chart & Reference \\
\hline 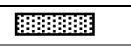 & [40] & 曹两曲 & [44] & (1:1:19 & [49] & प110 & [54] \\
\hline$\$ \otimes$ & [41] & एХ & [45] & 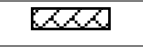 & [50] & 558 & [55] \\
\hline ๘ & [42] & $\mathbf{N D}$ & [47] & $\ldots \cdots$ & [51] & $\% \%$ & [56] \\
\hline Fins & [43] & ⿷匚⿳丨コ工卜도 & [48] & ए人 & [53] & & \\
\hline
\end{tabular}


Figure 21: Percentage of drag reduction by passive control methods.

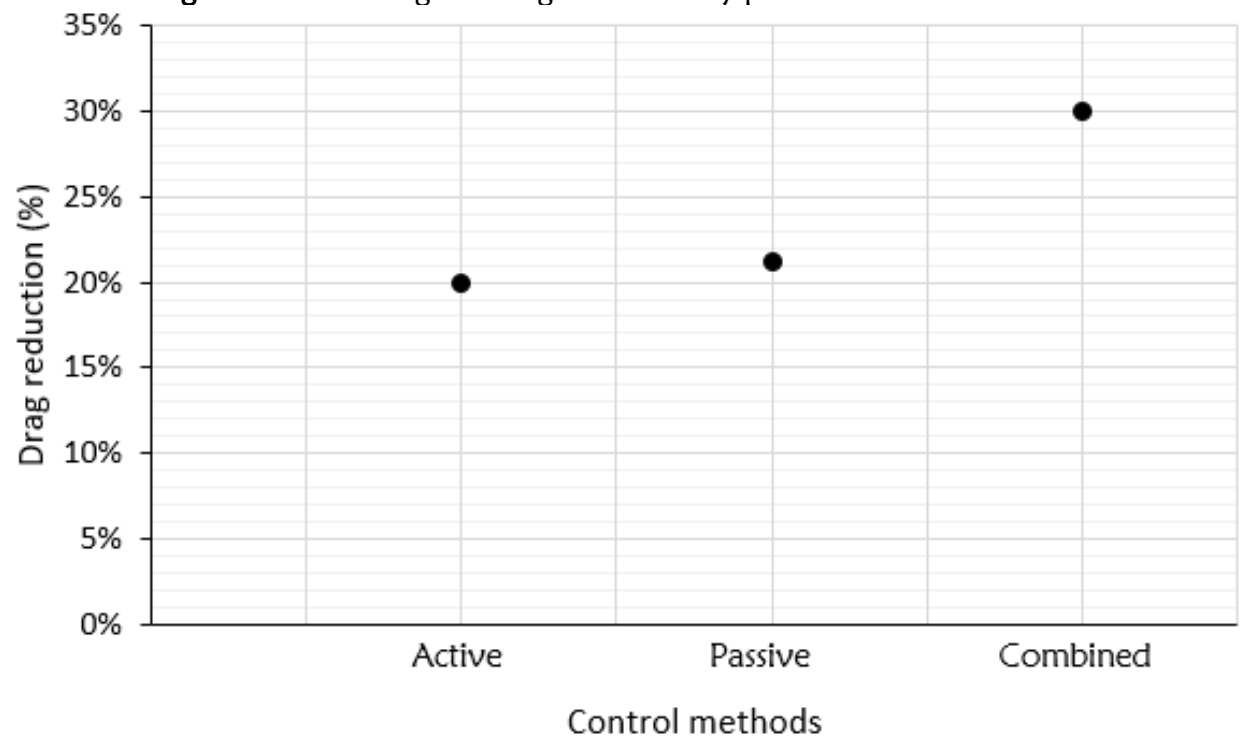

Figure 22: Maximum contribution of drag reduction for different control methods.

\section{CONCLUSIONS}

The vehicle aerodynamic drag is the main contributor to increase the fuel consumption which affects not only the world fuel reserves but also the environment. Thereby, reducing aerodynamic drag is the best solution for the greener world as well as for the fuel hike. Various flow control methods have been tested and validated numerically and experimentally of whose, most of the cases, simplified Ahmed body has been chosen. The major findings from the present review analysis may be summarized as follows:

1. Passive flow control methods employ the additional shape on the vehicle body which affect the flow field thus desirable impact on drag reduction. But these methods are sometimes added extra drag due to its shape. Besides their attachment on vehicle body are little bit complex.

2. On the other hand, active flow control systems come in action only when it is necessary to control the flow. Their attachments are little bit easier than passive flow control. Main disadvantage of active flow control is that these require power to come in action which increase power consumption of vehicles.

3. The research outcomes about the drag reduction of vehicle from these control methods have been discussed in this review paper. It is conjectured that the drag reduction for active, passive and combined control systems could be as much as $20 \%, 21.2 \%, 30 \%$, respectively.

\section{REFERENCES}

1. Wood, R. M. (2004). Impact of advanced aerodynamic technology on transportation energy consumption. SAE Technical Paper, 2004-01-1306.

2. Levin, J., \& Rikdal, R. (2011). Aerodynamic analysis of drag reduction devices on the underbody for SAAB 9-3 by using CFD. Master's Thesis in Automotive Engineering, Chalmers University of Technology, Sweden.

3. Zhang, C., Uddin, M., Robinson, C., \& Foster, L. (2017). Full vehicle CFD investigations on the influence of frontend configuration on radiator performance and cooling drag, Applied Thermal Engineering, 130, 1328-1340.

4. Regin, F. (2010). A Numerical Analysis on Air-cooling Performance of Passenger Cars. SAE Technical Paper, 201001-0554.

5. Aljure, D. E., Calafell, J., Baez, A., \& Oliva, A. (2018). Flow over a realistic car model: Wall modeled large eddy simulations assessment and unsteady effect. Journal of Wind Engineering \& Industrial Aerodynamics, 174, 225240.

6. Matsumoto, D., Haag, L., \& Indinger, T. (2017). Investigation of the unsteady external and underhood airflow of the driver model by dynamic mode decomposition methods. International Journal of Automotive Engineering. $8(2), 55-62$.

7. Tian, J., Zhang, Y., Zhu, H., \& Xiao, H. (2017). Aerodynamic drag reduction and flow control of Ahmed body with flaps. Advances in Mechanical Engineering, 9(7) 1-17.

8. Bansal, R., \& Sharma, R. B. (2014). Drag reduction of passenger car using add-on devices. Journal of Aerodynamics, 2014, 1-13. 
9. Mayer, W. \& Wickern, G. (2011). The new Audi A6/A7 family - aerodynamic development of different body types on one platform, SAE International Journal of Passenger Cars, 4(1), 197-206.

10. Cattafesta, L. N., \& Sheplak, M. (2011). Actuators for active flow control. Annual Review of Fluid Mechanics, 43(1), 247-272.

11. Kourta, A. \& Gilliéron, P. (2009). Impact of the automotive aerodynamic control on the economic issues. Journal of Applied Fluid Mechanics, 2(2), 69-75.

12. Altaf A., Omar, A., \& Asrar, W. (2014). Review of passive drag reduction techniques for bluff road vehicles, IIUM Engineering Journal, 15(1), 61-69.

13. Lee, S.W. (2018). Computational analysis of air jet wheel deflector for aerodynamic drag reduction of road vehicle. Microsystem Technologies, 1-11.

14. Hucho, W. H. \& Sovran, G. (1993). Aerodynamics of road vehicles. Annual Review of Fluid Mechanics, 25(1), 485-537.

15. Bellman, M., Agarwal, R., Naber, J., \& Chusak, L. (2010). Reducing energy consumption of ground vehicles by active flow control. ASME. Energy Sustainability, ASME 4th International Conference on Energy Sustainability, 1, 785-793.

16. Song, J., Yoshioka, S., Kato, T. \& Kohama, Y. (2006). Characteristics of flow behind a passenger vehicle. SAE Technical Paper Series, 2006-01-1030.

17. Hucho, W-H. (1990). Aerodynamics of road vehicles-from fluid mechanics to vehicle engineering, ButterworthHeinemann, Germany.

18. Kang, S. O., Jun, S. O., Park, H. I., Song, K. S., Kee, J. D., Kim, K. H., \& Lee, D. H. (2012). Actively translating a rear diffuser device for the aerodynamic drag reduction of a passenger car. International Journal of Automotive Technology, 13(4), 583-592.

19. Heinemann, T., Springer, M., Lienhart, H., Kniesburges, S., \& Becker, S. (2014). Active flow control on a 1: 4 car model, Exp Fluids 55: 1738, 1-11.

20. Mestiri, R., Ahmed-Bensoltane, A., Keirsbulck, L., Aloui, F., \& Labraga, L. (2014). Active flow control at the rear end of a generic car model using steady blowing. Journal of Applied Fluid Mechanics. 7(4), 565-571.

21. Roumeas, M., Gilli'eron, P., \& Kourta, A. (2009). Analysis and control of the near-wake flow over a square-back geometry. Computers \& Fluids 38(2009), 60-70.

22. Wassen, E. \& Thiele, F. (2008). Drag reduction for a generic car model using steady blowing, 4th Flow Control Conference, AIAA, 2008-3771.

23. Krentel D., Muminovic, R., Brunn, A., Nitsche, W., \& King, R. (2010). Application of active flow control on generic 3d car models. Active Flow Control II. Springer, 108, 223-239.

24. Wassen E., Eichinger S., \& Thiele F. (2010). Simulation of active drag reduction for a square-back vehicle. In: King R. (eds) Active Flow Control II. Notes on Numerical Fluid Mechanics and Multidisciplinary Design, 108, $241-255$.

25. Aubrun, S., Mcnally, J., Alvi, F, \& Kourta, A. (2011). Separation flow control on a generic ground vehicle using steady microjet arrays. Experiments in Fluids. Exp Fluids (2011) 51, 1177-1187.

26. Jonathan M., Erik F., Gregory R., Rajan K., Kunihiko T., Farrukh A., Yoshihiro Y., Kei, M. (2015). Drag reduction on a flat back ground vehicle with active flow control. Journal of Wind Engineering and Industrial Aerodynamics. 145 (2015), 292-303.

27. Park, H., Cho, J. H., Lee, J., Lee, D. H., \& Kim, K. H. (2013). Aerodynamic drag reduction of Ahmed model using synthetic jet array. SAE International Journal of Passenger Cars-Mechanical Systems, 6(1), 1-6.

28. Kourta, A., \& Leclerc, C. (2013). Characterization of synthetic jet actuation with application to ahmed body wake, Sensors and Actuators, A: Physical. 192, 13-26.

29. Tounsi, N., Mestiri, R., Keirsbulck, L., Oualli, H., Hanchi, S., \& Aloui, F. (2016). Experimental study of flow control on bluff body using piezoelectric actuators. Journal of Applied Fluid Mechanics. 9(2), 827-838.

30. Joseph, P., Amandolese, X., \& Aider, JL., (2012). Drag reduction on the $25^{\circ}$ slant angle Ahmed reference body using pulsed jets. Exp. Fluids, 52, 1169-1185.

31. Bideaux, E., Bobillier, P., Fournier, E., Gilliéron, P., El Hajem, M., Champagne, J.Y., Gilotte, P., \& Kourta, A. (2011). Drag reduction by pulsed jets on strongly unstructured wake: towards the square back control. International. Journal of Aerodynamics, 1(3/4), 282-298.

32. Gillieron, P., \& Kourta, A. (2013). Massive separation control analysis of the pulsed jet actuators effects, Mechanics \& Industry, 14, 441-445.

33. Kourta1, A. \& Gilliéron, P. (2009). Impact of the automotive aerodynamic control on the economic issues. Journal of Applied Fluid Mechanics, 2(2), 69-75.

34. Lehugeur, B., Gilliéron, P., \& Kourta, A. (2010). Experimental investigation on longitudinal vortex control over a dihedral bluff body. Exp. Fluids, 48, 33-48.

35. Wassen, E. \& Thiele, F. (2009). Road vehicle drag reduction by combined steady blowing and suction. AIAA 2009-4174.

36. Whiteman, J. (2016). Active Flow Control Schemes for Bluff Body Drag Reduction. MSc Engineering Thesis, Ohio State Univ.

37. Boucinha, V., Weber, R., \& Kourta, A. (2011). Drag reduction of a 3D bluff body using plasma actuators. International Journal of Aerodynamics, Vol. 1(3/4), 262-281. 
38. Shadmani, S., Nainiyan, S. M. M., Ghasemiasl, R., Mirzaei, M., \& Pouryoussefi, S. G. (2018). Experimental study of flow control over an Ahmed body using plasma actuator. Mechanics and Mechanical Engineering, 22(1), 239251.

39. Khalighi, B., Ho, J., Cooney, J., Neiswander, B., Corke, T., \& Han, T. (2016). Aerodynamic Drag Reduction Investigation for a Simplified Road Vehicle Using Plasma Flow Control. Proceedings of the ASME 2016 Fluids Engineering Division Summer Meeting.

40. Aider, JL., Beaudoin, J., \& Wesfreid, J. E. (2010). Drag and lift reduction of a 3D bluff-body using active vortex generators. Exp. Fluids, 48, 771-789.

41. Kim, I. \& Chen, H. (2010). Reduction of aerodynamic forces on a minivan by a pair of vortex generators of a pocket type. International Journal of Vehicle Design, 53(4), 300-316.

42. Pujals, G., Depardon, S., \& Cossu, C. (2010). Drag reduction of a 3D bluff body using coherent streamwise streaks. Exp. Fluids, 49, 1085-1094.

43. Krajnovic, S. (2014). LES exploration of passive flow control around an Ahmed body. Journal of Fluids Engineering, ASME Transaction, 136(12), 1-28.

44. Mazyan, W. I. (2013). Numerical simulations of drag-reducing devices for ground vehicles. MSc Engg. Thesis, American University.

45. Shankar, G. \& Devaradjane, G. (2018). Experimental and computational analysis on aerodynamic behavior of a car model with vortex generators at different yaw angles. Journal of Applied Fluid Mechanics, 11(1), 285-295.

46. Filip, G., Maki, K., Bachant, P., \& Lietz, R. (2018). Simulation of flow control devices in support of vehicle drag reduction. SAE Technical Paper, 2018-01-0713.

47. Kim, I., Chen, H., \& Shulze, R. (2006). A rear spoiler of a new type that reduces the aerodynamic forces on a mini-van," SAE Technical Paper 2006-01-1631.

48. Beaudoin, JF. \& Aider, JL. (2008). Drag and lift reduction of a 3D bluff body using flaps. Exp. Fluids, 44, 491 501.

49. Kim, D., Lee, H., Yi, W., \& Choi, H. (2016). A bio-inspired device for drag reduction on a three-dimensional model vehicle. Bioinspiration \& Biomimetics, 11(2), 1-13.

50. Tian, J., Zhang, Y., Zhu, H., \& Xiao, H. (2017). Aerodynamic drag reduction and flow control of Ahmed body with flaps. Advances in Mechanical Engineering, 9(7), 1-17.

51. Marklund, J., Lofdahl, L., Danielsson, H., \& Olsson, G. (2013). Performance of an automotive under-body diffuser applied to a sedan and a wagon vehicle. SAE International Journal of Passenger Cars, 6(1), 293-307.

52. Unni, T. P. A. (2017). Numerical investigation on aerodynamic effects of vanes and flaps on automotive underbody diffusers. SAE Technical Paper, 2017-01-2163.

53. Cho, J., Kim, TK., Kim, KH., \&Yee, K. (2017). Comparative investigation on the aerodynamic effects of combined use of underbody drag reduction devices applied to real sedan. International Journal of Automotive Technology, 18(6), 959-97.

54. Song, K.S., Kang, S.O., Jun, S.O., Park, H. I., Kee, J. D., Kim, K. H., \& Lee, D. H. (2012). Aerodynamic design optimization of rear body shapes of a sedan for drag reduction. International Journal of Automotive Technology, 13(6), 905-914.

55. Hu, X., Yang, B., Lei, Y., Wang, J., Li, X., Liao, L., \& Xu, T. (2016). Automotive shape optimization using the radial basis function model based on a parametric surface grid. Proceeding of International Mechanical Engineering, Part D: Journal of Automobile Engineering, 1-14.

56. Wang, Y., Wu, C., Tan, G., \& Deng, Y. (2016). Reduction in the aerodynamic drag around a generic vehicle by using a non-smooth surface. Proceeding of International Mechanical Engineering, Part D: Journal of Automobile Engineering, 1-15.

57. Bruneau, CH., Creusé, E., Depeyras, D., Gilliéron, P., \& Mortazavi, I. (2010). Coupling active and passive techniques to control the flow past the square back Ahmed body. Computers \& Fluids, 39 (2010), 1875-1892. 